Internist 2015 $\cdot 56: 737-738$

DOI 10.1007/s00108-015-3669-0

Online publiziert: 17. Juni 2015

(c) Springer-Verlag Berlin Heidelberg 2015

H. Haller ${ }^{1}$ - E. Märker-Hermann ${ }^{2}$

${ }^{1}$ Abteilung Nephrologie, Medizinische Hochschule Hannover

${ }^{2}$ Klinik für Innere Medizin IV, HSK Wilhelm Fresenius Klinik, Wiesbaden

\title{
Störungen des Elektrolyt- und Säure-Basen-Haushalts
}

\section{Einfach diagnostizierbare Zustände und komplexe Situationen}

breiten Palette von Artikeln dazu beigetragen.

Im Beitrag von D. Müller aus Berlin werden Elektrolytstörungen als erstes Merkmal monogenetischer Erkrankungen beschrieben. Störungen der Kaliumund Natrium/Wasser-Regulation sind bei Vorliegen anderer Befunde wie Hypertonie oft erstes Merkmal einer zugrunde liegenden genetischen Störung. Diese Störungen sind selten. Deshalb ist es wichtig, die notwendigen diagnostischen Maßnahmen bei Verdacht auf solche Erkrankungen zu überblicken.

\section{) Auch die Therapie von Elektrolytstörungen richtet sich nach der pathophysio- logischen Ursache}

Im zweiten Beitrag thematisiert R. Kettritz, ebenfalls aus Berlin, die iatrogen verursachten Elektrolytstörungen, so etwa beim Einsatz von Diuretika und anderen Medikamenten. Der Autor schildert an Beispielen, welche Medikamente bzw. Medikamentenkombinationen bei Vorliegen einer Elektrolytstörung zu berücksichtigen sind und als Ursache ausgeschlossen werden müssen.

Im dritten Beitrag beschäftigt sich B. Schmidt aus Hannover mit Elektrolytstörungen in der akuten Situation einer
Aufnahmestation. Hier werden die meisten Elektrolytstörungen zuerst festgestellt. In seinem Beitrag unterscheidet B. Schmidt zwischen den akuten Notfallsituationen und den Elektrolytstörungen, die eher chronisch behandelt werden müssen.

Die Therapie der Elektrolytstörungen ist Gegenstand der folgenden Beiträge. J. Hensen aus Hannover erörtert zuerst die Rolle von Vasopressinantagonisten in der Behandlung der Hyponatriämie. Vor mehr als 5 Jahren wurden diese Medikamente in die Therapie eingeführt. Die Nebenwirkungsrate ist nicht niedrig. J. Hensen setzt sich mit den therapeutischen Einsatzmöglichkeiten unter Berücksichtigung der Probleme in der Therapie mit Vasopressinantagonisten auseinander.

P. Kümpers aus Münster diskutiert in seinem Beitrag die Rolle der Volumensubstitution bei Elektrolytstörungen. Sein Beitrag befasst sich mit der Frage, welche und wie viele Ersatzlösungen bei der Volumentherapie eingesetzt werden sollen.

》) Eine Reihe von Kaliumsenkern steht kurz vor der Zulassung

R. Schmitt aus Hannover beschäftigt sich mit einer neuen Klasse von Medikamenten, den sog. Kaliumsenkern. Eine Rei- 
he von Wirkstoffen aus dieser Gruppe steht kurz vor der Zulassung. Der Autor erörtert die therapeutischen Möglichkeiten und die Probleme, welche diese neuen Therapeutika mit sich bringen werden.

Im letzten Beitrag behandelt J. Hoyer aus Marburg den Einfluss von Natrium auf den Blutdruck. Eine jahrzehntelange Kontroverse zum Stellenwert von Natriumchlorid in der Pathogenese der Hypertonie und als Risikofaktor kardiovaskulärer Erkrankungen wird von ihm zusammengefasst. Der Autor beleuchtet den derzeitigen Wissensstand bezüglich des Zusammenhangs von Natrium und Hypertonie.

Wir hoffen, Ihr Interesse geweckt zu haben, und wünschen Ihnen eine interessante Lektüre.

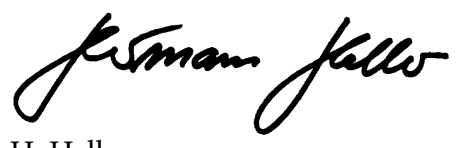

H. Haller

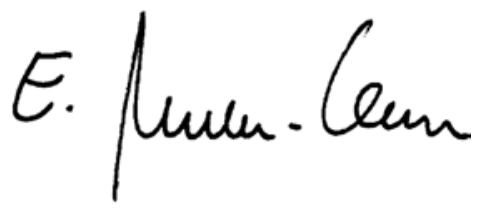

E. Märker-Hermann

\section{Korrespondenzadresse}

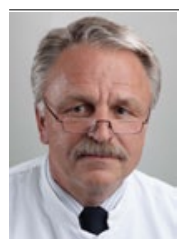

Prof. Dr. H. Haller

Abteilung Nephrologie,

Medizinische Hochschule

Hannover

Carl-Neuberg-Str. 1

30625 Hannover

nephrologie@mh-hannover.de

Interessenkonflikt. H. Haller und E. Märker-Hermann geben an, dass kein Interessenkonflikt besteht.

\section{HIV/STI-Prävention: Preisträger des Sirius2015 ausgezeichnet}

Am 5. Mai 2015 wurde in Berlin der

Sirius2015, der Preis des Bundeswett-

bewerbs zur HIV/STI-Prävention,

verliehen.

Zehn Projekte wurden für besonders innovative, bedarfsgerechte und erfolgversprechende Präventionsansätze zu HIV und anderen sexuell übertragbaren Infektionen (STI) ausgezeichnet.

Während die Präventionsarbeit zu HIV in Deutschland sehr erfolgreich ist, sind andere sexuell übertragbare Infektionen hingegen weiterhin auf dem Vormarsch. STI sind oft noch wenig bekannt, die Gefahren werden häufig unterschätzt. Sie können das Risiko einer HIV-Infektion deutlich erhöhen, stellen aber auch ein eigenständiges Gesundheitsrisiko dar. Beispielsweise kann eine HPV-In-

fektion eine spätere Gebärmutterhalskrebserkrankung auslösen und Chlamydien können unbehandelt zu Unfruchtbarkeit führen. Der Bundeswettbewerb Sirius2015 unterstützt gezielt Strategien zur Verhütung einer Ansteckung und zur frühen Erkennung und Behandlung bereits erfolgter Infektionen.

Zur Beteiligung am Sirius2015 waren bundesweit Beratungsstellen, Vereine, Schulen, Universitäten, Institutionen, Verbände und kirchliche Einrichtungen eingeladen. Über die Vergabe der Preise entschied eine praxiserfahrene Jury mit hoher Expertise in der HIVund STI-Prävention.

Der Sirius2015 ist ein gemeinsames Projekt des Bundesministeriums für Gesundheit (BMG) und der Bundeszentrale für gesundheitliche Aufklärung (BZgA) zusammen mit dem Verband der Privaten Krankenversicherung e. V. (PKV). Mit Preisgeldern von insgesamt über 100.000 Euro werden vor allem Projekte gefördert, die sich besonders an schwer erreichbare oder benachteiligte Gruppen richten. Über 50 Projekte hatten sich beworben.

Die Preisträger des Sirius2015 sind:

- Ragazzamobil: Mobile Gesundheitsprävention für drogenkonsumierende Sexarbeiterinnen, ragazza e.V., Hamburg

- Hein \& Fietes STI Studio: Visuelle Aufklärung und Prävention als ehrenamtliche Initiative von Hein \& Fiete, Prävention e.V., Hamburg
- Wir für alle: Gemeinsam gegen AIDS bei afrikanischen Flüchtlingen, ein kultursensibles Präventionsprojekt bei afrikanischen Flüchtlingen vom Verein Gemeinsamer Arm gegen AIDS e.V., Nürnberg

- Gesund in Haft: Ein Videoprojekt der Münchner AIDS-Hilfe e.V. im Rahmen eines studentischen Projektes der Katholischen Stiftungsfachhochschule, München

- Sex in the City! Eine sexualpädagogische Kiezrallye in Schöneberg vom Verein MannO-Meter e.V., Berlin, in Kooperation mit der Berliner AIDS-Hilfe e.V., pro familia Berlin und LSVD Berlin Brandenburg e. V.

- Socke \& Schuss: mit Herzenslust bis in die Puppen, humorvolle Bildsprache und Dialoge mit Handpuppen unter der Leitung der AIDSHilfe NRW e.V., Köln

Diese sechs Projekte erhalten für Auf- und Ausbau oder die Fortsetzung ihrer Arbeit jeweils ein Preisgeld zwischen 15.000 Euro und 20.000 Euro. Darüber hinaus hat die Jury vier weitere Projekte mit einem Anerkennungspreis in Höhe von jeweils 2.500 Euro ausgezeichnet:

Quelle: Bundeszentrale für gesundheitliche Aufklärung, www.bzga.de 